Pacific Journal of Mathematics

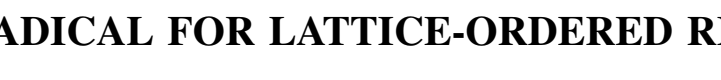




\title{
A RADICAL FOR LATTICE-ORDERED RINGS
}

\author{
J. E. DIEM
}

The main result of this paper states that for a latticeordered ring ( $l$-ring) $A$ with no nonzero nilpotent $l$-ideals the following are equivalent: (i) $A$ is an $f$-ring; (ii) $A$ is a subdirect union of totally-ordered rings with no nonzero divisors of zero; (iii) $x^{+} x^{-}=0$ for all $x \in A$; (iv) $x^{+} a x^{-}=0$ for all $x, a \in A$; and $(\mathbf{v}) a(b \vee c)=a b \vee a c$ and $(b \vee c) a=b a \vee c a$ for all $a, b, c \in A$ with $a \geqq 0$. In particular, the equivalence of (i) and (iii) implies that an $l$-ring which has an identity that is a weak order unit and which has no nonzero nilpotent $l$ ideals is necessarily an $f$-ring.

The basic tool in our considerations is the notion of prime $l$-ideal. Specifically, call a proper $l$-ideal $P$ of an $l$-ring $A$ prime if $J \subseteq P$ or $J \subseteq P$ wherever $I$ and $J$ are $l$-ideals of $A$ with $I J \subseteq P$. Various conditions are obtained on $A$, each of which forces $A$ modulo every prime $l$-ideal to be totally-ordered with no nonzero divisors of zero. Moreover the relationship between the join of all the nilpotent $l$-ideals of $A$ and the intersection of all the prime $l$-ideals of $A$ is investigated in order to obtain the theorem mentioned above.

The $P$-radical of an $l$-ring $A$ is the intersection of all the prime $l$-ideals of $A$. In $\S 2$ the general theory of the $P$-radical is considered. The results here are analogous to ring theoretic results found in McCoy [4] and Jacobson [2] (Chapter VIII).

In $\S 3$ the general theory of the $P$-radical which is more or less independent of the order structure is tied together with the order. Specifically we investigate the relationship between the $P$-radical and the join of all of the nilpotent $l$-ideals for various classes of $l$-rings.

$\$ 4$ contains a proof of the theorem mentioned above.

2. Prime $l$-ideals and the $P$-radical. The results of this section are analogous to ring theoretic results found in McCoy [4] and Jacobson [2] (Chapter VIII). Consequently after proving a few of the results in detail, we sketch proofs indicating the idiosynrasies they take on in l-rings and note the analogous result in McCoy or Jacobson.

The reader is referred to Birkhoff and Pierce [1] and Johnson [3] for the general theory of $l$-rings. Our notation is the same as Johnson [3]. Also, the word $l$-ideal, unmodified means proper $l$-ideal.

Definition 2.1. (i) An $l$-ideal $P$ of an $l$-ring $A$ is prime if $I \subseteq P$ or $J \subseteq P$ whenever $I$ and $J$ are $I$-ideals of $A$ with $I J \subseteq P$.

(ii) A nonzero $l$-ring $A$ is prime if $\{0\}$ is a prime $l$-ideal. 
(iii) A nonzero $l$-ring $A$ is an $l$-domain if $A^{+} \backslash\{0\}$ is closed under multiplication.

REMARK. If $I$ and $J$ are $l$-ideals of an $l$-ring $A$, then $I J$ denotes the ring theoretic product of the ideals $I$ and $J$. Note that $I J$ is not, in general, an $l$-ideal. We can "make $I J$ into an $\ell$-ideal" by forming $\langle I J\rangle$, the smallest $l$-ideal containing $I J$. Birkhoff and Pierce [1] have denoted this by $I \cdot J$ and called it the $l$-product of $I$ and $J$. As we shall have ocassion to use the notation $\langle S\rangle$ for the $l$-ideal generated by a subset $S$ of an $l$-ring $A$, we use the notation $\langle I J\rangle$ for the $l$ product of two $l$-ideals $I$ and $J$. Note that if $I, J$, and $P$ are $l$-ideals of $A$, then $I J \subseteq P$ if and only if $\langle I J\rangle \subseteq P$; and hence the definition of prime $l$-ideal is independent of the choice of $I J$ or $\langle I J\rangle$.

To set the situation we note that a prime $l$-ideal need not be prime as a ring ideal. In fact, a prime $l$-ideal of an archimedean commutative $l$-ring in which the square of every element is positive need not be prime as a ring ideal (See 2.3 below.). However, Johnson [3] has shown.

Theorem 2.2. Let $A$ be an $f$-ring, ${ }^{1}$ and let $P$ be an l-ideal of A. Then the following are equivalent:

(i) $A / P$ is totally-ordered with no nonzero divisors of zero;

(ii) $P$ is prime as a ring ideal; and

(iii) $P$ is a prime l-ideal.

In $\S 4$ we generalize 2.2 to several classes of $l$-rings each of which properly contains the class of $f$-rings.

EXAMPLE 2.3. A prime $l$-ideal of an archimedean commutative $l$-ring in which the square of every element is positive which is not prime as a ring ideal.

Let $S$ be the semigroup consisting of two elements $a$ and $b$ with multiplication $a b=b a=a^{2}=b^{2}=a$, and let $R(S)$ denote the semigroup ring on $S$ with real coefficients. Make $R(S)$ into an archimedean commutative $l$-ring by decreeing that $\alpha a+\beta b \geqq 0$ if $\alpha \geqq 0$ and $\beta \geqq 0$ where $\alpha$ and $\beta$ are real numbers. Then the square of every element of $R(S)$ is positive since $(\alpha a+\beta b)^{2}=(\alpha+\beta)^{2} a$. Now, $\{0\}$ is not prime as a ring ideal since $(a-b)^{2}=0$. However, it is easy to see that $R(S)$ is an $l$-domain, and hence $\{0\}$ is a prime $l$-ideal by the next result.

\subsection{If $P$ is l-ideal of an l-ring $A$ such that $A^{+} \backslash P$ is closed}

${ }^{1}$ An $f$-ring is an $l$-ring in which $a \wedge b=0$ and $c \geqq 0$ imply $c a \wedge b=0$ and $a c \wedge b=0$. In [1] Birkhoff and Pierce showed that the class of $f$-rings is identical with the class of subdirect unions of totally-ordered rings. 
under multiplication, then $P$ is a prime l-ideal. The converse holds is $A$ is commutative.

Proof. First suppose that $I$ and $J$ are $l$-ideals of $A$ with $I J \subseteq P$. If $I$ is not contained in $P$, then there is a non-zero positive element $a \in I \backslash P$. Let $b$ be a positive element of $J$. Then $a b \in I J \subseteq P$, so that $b \in P$ since $a \notin P$. It follows that $J \subseteq P$.

Now suppose that $A$ is commutative, $P$ is a prime $l$-ideal of $A$, and $a_{1}, a_{2} \in A^{+}$with $a_{1} a_{2} \in P$. Then $\left\langle a_{1} a_{2}\right\rangle \subseteq P$. Let $z_{i} \in\left\langle a_{i}\right\rangle, i=1,2$. Then $\left|z_{i}\right| \leqq n_{i} a_{i}+r_{i} a_{i}(i=1,2$.$) for suitable r_{i} \in A^{+}$and suitable nonnegative integers $n_{i}$. Thus

$$
\left|z_{1} z_{2}\right| \leqq\left|z_{1}\right|\left|z_{2}\right| \leqq\left(n_{1} a_{1}+r_{1} a_{1}\right)\left(n_{2} a_{2}+r_{2} a_{2}\right)
$$

which belongs to $P$ since $A$ is commutative and $\left\langle a_{1} a_{2}\right\rangle \subseteq P$. It follows that $\left.\left\langle a_{1}\right\rangle a_{2}\right\rangle \subseteq P$; and hence either $a_{1} \in P$ or $a_{2} \in P$.

The following characterization of prime $l$-ideals will be used repeatedly in the sequel.

2.5. An l-ideal $P$ of an l-ring $A$ is prime if and only if $a, b \in A^{+}$ and $a A^{+} b \subseteq P$ imply $a \in P$ or $b \in P$.

Proof. Necessity. From $a A^{+} b \subseteq P$ it follows that

$$
\left\langle A^{+} a A^{+} \backslash A^{+} b A^{+}\right\rangle \subseteq P .
$$

Thus either $\left\langle A^{+} a A^{+}\right\rangle \subseteq P$ or $\left\langle A^{+} b A^{+}\right\rangle \subseteq P$. Suppose that $\left\langle A^{+} a A^{+}\right\rangle \leqq P$. Then $\langle a\rangle^{3} \subseteq P$, and hence $\langle\langle a\rangle\langle a\rangle\rangle\langle a\rangle \subseteq P$. Thus either $\langle a\rangle^{2} \subseteq P$ or $\langle a\rangle \subseteq P$. In either case we have that $a \in P$.

Sufficiency. If $I$ and $J$ are $l$-ideals of $A$ which are not contained in $P$, then there is an $a \in I^{+} \backslash P$ and a $b \in J^{+} \backslash P$. If $I J \subseteq P$, then $a A^{+} b \subseteq I J \subseteq P$; so that $a \in P$ or $b \in P$. Since this contradicts the choice of $a$ and $b, I J$ is not contained in $P$; and we are done.

Note that 2.5 says that an $l$-ideal $P$ of an $l$-ring $A$ is prime if and only if $A^{+} \backslash P$ is an $m$-system in the sense of

Definition 2.6. A nonempty subset $M$ of an $l$-ring $A$ is an $m$ system if each element of $M$ is positive and if for $a, b \in M$ there is an $x \in A^{+}$such that $a x b \in M$.

Note that nonempty subset $S$ of $A^{+}$which is closed under multiplication is an $m$-system since $a a b \in S$ whenever $a, b \in S$.

The next result, as did the proceeding, has its analogue in [4].

2.7. Let $M$ be an $m$-system of an l-ring $A$, and let $I$ be an $l$ - 
ideal of $A$ that does not meet $M$. Then $I$ is contained in a prime l-ideal that does not meet $M$.

Proof. The existence of an $l$-ideal $P$ of $A$ which is maximal with respect to the property of not meeting $I$ is guaranteed by Zorn's Lemma. We show that $P$ is prime. The proof of this is an in [4] (Lemma 4) once one knows that the $l$-ideal generated by $P$ and a positive element $a$ of $A$ not in $P$ is $\{z \in A:|z| \leqq p+n a+r a+s a+t a v$ where $r, s, t, v \in A^{+}, p \in P^{+}$, and $n$ is a nonnegative integer $\}$.

Definition 2.8. The $P$-radical, $P(A)$, of an $l$-ring $A$ is the intersection of all of the prime $l$-ideals of $A$.

Recall that the $l$-radical of an $l$-ring $A$ is the set $N(A)=\{a \in A$ : there is a positive integer $n=n(a)$ such that

$$
x_{0}|a| x_{1}|a| x_{2} \cdots x_{n-1}|a| x_{n}=0
$$

for all $\left.x_{0}, x_{1}, x_{2}, \cdots, x_{n} \in A\right\}$ ([1], p. 45.) If $A$ is comutative, then $N(A)=\{a \in A:|a|$ is nilpotent $\}$ ([1], Corollary 1, p. 45). Moreover, for an arbitrary $l$-ring $A, N(A)$ is the join of all of the nilpotent $l$ ideals of $A$ ([1], Th. 5).

Now suppose that $a \in A$ is not nilpotent. Then since $0<\left|a^{n}\right| \leqq$ $|a|^{n}$ for all $n,|a|$ is not nilpotent. Thus, by 2.7 , there is a prime $l$-ideal $P$ of $A$ not meeting the $m$-system $\left\{|a|,|a|^{2}, \cdots,|a|^{n}, \cdots\right\}$. It follows that $a$ does not belong to $P(A)$, and hence every element of $P(A)$ is nilpotent. Now note that every prime $l$-ideal of $A$ contains every nilpotent $l$-ideal of $A$, and hence we have

2.9. The P-radical of an l-ring $A$ is a nil l-ideal of $A$ containing the l-radical of $A$.

The proof of the next result is as in [4] (Theorem 5).

2.10. If $A$ is an l-ring, then $P(A / P(A))$ is zero.

The next result is useful in relating the $l$-radical to the $P$-radical.

2.11. Let $I$ be an l-ideal of an l-ring $A$ such that $N(A / I)$ is zero, and let $J$ be an l-ideal of $A$ properly containing $I$. Then there is a prime l-ideal $P$ of $A$ containing $I$ but but not containing $J$.

Proof. (After Jacobson, [2], p. 196) Choose $a_{0} \in J^{+} \backslash I$. Then since $N(A / I)$ is zero, $A / I$ has no nonzero nilpotent $l$-ideals; and hence $\left\langle a_{0}\right\rangle^{k}$ is not contained in $I$ for any positive integer $k$. Now, $\left\langle A^{+} a_{0} A^{+}\right\rangle^{2}$ is 
not contained in $I$ since $\left\langle a_{0}\right\rangle^{3} \cong\left\langle A^{+} a_{0} A^{+}\right\rangle$and $\left\langle a_{0}\right\rangle^{6}$ is not contained in $I$. Now suppose that $a_{0} b a_{0} \in I$ for all $b \in A^{+}$. Then for $z \in\left\langle A^{+} a_{0} A^{+}\right\rangle^{2}$, there are $x_{i}, \mathrm{y}_{i} \in\left\langle A^{+} a_{0} A^{+}\right\rangle$and $t_{i}, u_{i}, v_{i}, w_{i}, \in A^{+}$such that

$$
|z| \leqq \sum_{i=1}^{n}\left|x_{i} \| y_{i}\right| \leqq \sum_{i=1}^{n}\left(t_{i} a_{0} u_{i}\right)\left(v_{i} a_{0} w_{i}\right) .
$$

But $a_{0} u_{i} v_{i} a_{0} \in I^{+}$, so that $z \in I$. Consequently there is a $b_{0} \in A^{+}$such that $a_{1}=a_{0} b_{0} a_{0} \in J^{+} \backslash I$. Similarly, there is a $b_{1} \in A^{+}$such that $a_{2}=$ $a_{1} b_{1} a_{1} \in J^{\dagger} \backslash I$. Containing inductively, we obtain two sequences: $\left\{a_{i}\right\}_{i=0}^{\infty} \subseteq$ $J^{+} \backslash I$ and $\left\{b_{i}\right\}_{i=0}^{\infty} \subseteq A^{+}$such that $a_{n}=a_{n-1} b_{n-1} a_{n-1} \in J^{+} \backslash I$ for all $n \geqq 1$. It follows that $\left\{a_{i}\right\}_{i=0}^{\infty}$ is an $m$-system that does not meet $I$. By 2.7 there is a prime $l$-ideal $P$ of $A$ containing $I$ that does not meet $\left\{a_{i}\right\}_{i=0}^{\infty}$. Since $a_{i} \in J$ for $i \geqq 0$, we know that $J$ is not contained in $P$; and hence $P$ is as desired.

2.12. If $A$ is an l-ring, then $P(A)=\bigcap\{I: I$ is an l-ideal of $A$ and $N(A / I)$ is zero\}.

Proof. Let $\mathscr{L}(A)=\bigcap\{I: I$ is an $l$-ideal of $A$ and $N(A \backslash I)$ is zero $\}$. If $P$ is a prime $l$-ideal of $A$, then $N(A \backslash P) \subseteq P(A \backslash P)=\{0\}$. Thus $\mathscr{L}(A) \subseteq P(A)$.

Now let $J / \mathscr{L}(A)$ be a nilpotent $l$-ideal of $A / \mathscr{L}(A)$, and let $I$ be an $l$-ideal of $A$ such that $N(A / I)$ is zero. Then $J^{n} \subseteq \mathscr{L}(A)$ for some positive integer $n$; and since $\mathscr{L}(A) \subseteq I$, we know that $J^{n} \subseteq I$. It follows that $\langle I+J\rangle / I$ is a nilpotent $l$-ideal of $A / I$. Since $N(A / I)$ is zero, it follows that $J \subseteq I$. Thus $J \subseteq \mathscr{L}(A)$, so that $N(A / \mathscr{L}(A)$ ) is zero. Now if $\mathscr{L}(A)$ is properly contained in $P(A)$, then, by 2.11 there is a prime $l$-ideal containing $\mathscr{L}(A)$ but not containing $P(A)$. Since this contradicts the definition of $P(A), \mathscr{L}(A)=P(A)$.

2.13. If $A$ is an l-ring, the $N(A / N(A))$ is zero if and only if if $N(A)=P(A)$. Hence $N(A)$ is zero if and only if $P(A)$ is zero.

Proof. If $N(A / N(A))$ is zero, then $P(A)=\bigcap\{I: I$ is an $l$-ideal of $A$ and $N(A / I)$ is zero $\leqq N(A) \subseteq P(A)$.

If $N(A)=P(A)$, then $N(A / N(A))=N(A / P(A)) \subseteq P(A / P(A))$ which is zero.

The next result has its analogue in [4] (Theorem 6). It will be used in $\S 4$ to obtain the theorem mentioned in the introduction.

2.14. An l-ring $A$ has zero l-radical if and only if it is a subdirect union of prime l-rings. 
Proof. The proof is immediate from 2.13.

The remaining results of this section will be useful in the next section where we determine various classes of $l$-rings for which the $P$-radical equals the $l$-radical.

2.15. If $A$ is an l-ring, then $P(A)=\{a \in A$ : any $m$-system containing $|a|$ contains 0 .

Proof. Suppose that there is an $m$-system $M$ containing $|a|$ that does not contain 0 . Then, by 2.7 , there is a prime $l$-ideal $P$ of $A$ that does not meet $M$. Thus $|a|$ does not belong to $P$, and it follows that $a$ does not belong to $P(A)$.

Conversely, let $a \in A$ be such that any $m$-system containing $|a|$ contains 0 , and let $P$ be a prime $l$-ideal of $A$. If $a$ does not belong to $P$, then $A^{+} \backslash P$ is an $m$-system containing $|a|$. Thus $0 \in A^{+} \backslash P$ which is clearly impossible. Hence $a \in P(A)$.

2.16. If $A$ is an l-ring, then $N(A)=\{a \in A$ : there is a positive integer $n=n(a)$ such that $(x|a|)^{n} x=0$ for all $\left.x \in A^{+}\right\}$.

Proof. It is clear from the definition of $N(A)$ that if $a \in N(A)$, then there is a positive integer $n$ such that $(x|a|)^{n} x=0$ for all $x \in A^{+}$.

Conversely, suppose that there is a positive integer $n$ such that $(x|a|)^{n} x=0$ for all $x \in A^{+}$, and let $x_{0}, x_{1}, \cdots, x_{n} \in A^{+}$. Then, since $x=x_{0} \vee x_{1} \vee \cdots \vee x_{n} \geqq x_{i}$ for all $i=0,1, \cdots, n$, it follows that $0=(x|a|)^{n} x \geqq x_{0}|a| x_{1} \cdots x_{n-1}|a| x_{n} \geqq 0$. Since every element of $A$ is the difference of two positive elements, the result follows.

2.17. If $I$ is a right (resectively, left) l-ideal of an l-ring $A$, then $P(I)=P(A) \cap I$.

Proof. Let $a \in P(I)$ and let $M$ be an $m$-system in $A$ containing $|a|$. We show that $M \cap I$ is an $m$-system in $I$. Let $x, y \in M \cap I$. Then there is a $z \in A^{+}$such $x z y \in M \cap I$. Again there is a $z_{1} \in A^{+}$such that $x z y z_{1} x z y \in M \cap I$. But $z y z_{1} x z \in I^{+}$since $I$ is a right (respectively, left) $l$-ideal; hence $M \cap I$ is an $m$-system in $I$. By $2.15,0 \in M \cap I$ since $|a| \in M \cap I$ and $a \in P(I)$. Again, by 2.15, it follows that $a \in P(A) \cap I$.

Conversely, let $a \in P(A) \cap I$, and let $M$ be an $m$-system in I containing $|a|$. Then $M$ is an $m$-system in $A$ containing $|a|$. By 2.15, $M$ contains 0 ; and hence $a \in P(I)$.

2.18. If $I$ is a right (respectively, left) l-ideal of an l-ring $A$, 
then $N(I)=N(A) \cap I$.

Proof. If $a \in N(I)$, then, by 2.16, there is a positive integer $n$ such that $(x|a|)^{n} x=0$ for all $x \in I^{+}$. But for $y \in A^{+}$we know that $y|a| y \in I^{+}$, and hence $0=(y|a \| y| x)^{n} y=(y|a|)^{2 n+1} y$; so that

$$
y \in N(A) \cap I
$$

by 2.16. That $N(A) \cap I \subseteq N(I)$ is clear from the definition of $N(A)$.

3. The P-radical equals the $l$-radical. Birkhoff and Pierce ([1], p. 45, Example 8) have given an example of an $l$-ring $A$ such that $N(A / N(A))$ is not zero. By 2.13 , the $l$-radical of such an l-ring is properly contained in its $P$-radical. However, there are many $l$-rings for which the $l$-radical is equal to the $P$-radical. In this section we identify some of them and prove some results about $l$-rings in which the square of every element is positive.

THEOREM 3.1. If $A$ is an l-ring which is commutative, or satisfies either the ascending or descending chain condition on l-ideals, or is an f-ring, then $N(A)=P(A)$.

Proof. Birkhoff and Pierce ([1], p. 46, Corollary 4; and [1], p. 63, Corollary 1 ) have shown that if an $l$-ring $A$ is commutative, or satisfies either the ascending or descending chain condition on $l$-ideals, or is an $f$-ring, then $N(A / N(A))$ is zero. The result follows from 2.13.

Corollary 3.2. If $A$ is an l-ring, and if $P(A)$ is commutative, or satisfies either the ascending or descending chain condition on $l$-ideals, or is an f-ring, then $N(A)=P(A)$.

Proof. Using $2.9,2.17,2.18$, and 3.1 , we have

$$
N(A)=N(A) \cap P(A)=N(P(A))=P(P(A))=P(A) \cap P(A)=P(A) .
$$

In [1] Birkhoff and Pierce show that is $A$ is an $l$-ring with an identity element 1 that is a weak order unit ${ }^{2}$, then every nilpotent of $A$ is, in absolutive value, $\leqq 1$. We generalize this result to

Theorem 3.3. Let $A$ be an l-ring with an identity element 1, and suppose that the square of every element of $A$ is positive. Then each nilpotent $x$ of $A$ is, in absolute value, $\leqq 1$.

Proof. (We are indebted to the referee for this proof.) The

${ }^{2}$ A positive element $e$ of an $l$-ring $A$ is a weak order unit if $e \wedge x=0$ and $x \in A$ imply $x=0$. 
proof is by induction on the nilpotency index $k$ of $x$. For $k=1$ the result is trivial. For $k \geqq 1$ nilpotency index of $x^{2}$ is less than $k$. Thus $x^{2}=\left|x^{2}\right| \leqq 1$. Since $0 \leqq(x-1)^{2}=x^{2}-2 x+1$ and $0 \leqq(x+1)^{2}=$ $x^{2}+2 x+1$, we have that $-\left(1+x^{2}\right) \leqq 2 x \leqq 1+x^{2}$. Thus $2|x|=$ $|2 x| \leqq 1+x^{2} \leqq 2$. 1 , and hence $|x| \leqq 1$.

COROLlary 3.4. Let $A$ be an l-ring with an identity element 1 , and suppose that the square of every element of $A$ is positive. Then $N(A)=P(A)$.

Proof. By 3.3, $B(A)=\{x \in A:|x| \leqq n 1$ for some positive integer $n$ \} contains all of the nilpotents of $A$, and hence it contains $P(A)$. Now, Birkhoff and Pierce [1] have shown (and it is easy to see) that $B(A)$ is a sub-l-ring of $A$ which is an $f$-ring. Consequently $P(A)$ is a sub-f-ring of $A$, so-that, by $3.2, N(A)=P(A)$.

We now turn our attention to finding a sufficient condition for the $P$-radical of an $l$-ring $A$ in which the square of every element is positive to be equal to $\{x \in A ;|x|$ is nilpotent $\}$.

LEMmA 3.5. Let $A$ be an l-ring in which the square of every element is positive. Then for $a, b \in A^{+}$with $a^{2}=b^{2}=0$, we have that $a b=b a=0$.

Proof. Since $a b, b a$, and $(a-b)^{2}$ are positive, we know that $0 \leqq(a-b)^{2}=-b a-a b \leqq 0$. Thus $a b+b a=0$, and the lemma follows.

Lemma 3.6. Let $A$ be a prime l-ring in which the square of every element is positive. Then $A$ is an l-domain if and only if $a, b \in A, a \wedge b=0$, and $a b=0$ imply $b a=0$.

Proof. Necessity is clear since if $A$ is an $l$-domain and $a, b \in A^{+}$ are such that $a b=0$, then either $a=0$ or $b=0$.

Conversely, we first show that $A$ has no nonzero positive nilpotents of index 2. Suppose that $a \in A^{+}$and $a^{2}=0$, and let $z \in A^{+}$. We will show that $a z a=0$. There are three cases.

1. $0 \leqq z a \leqq a z$. Then $0 \leqq a z a \leqq a^{2} z=0$, so that $a z a=0$.

2. $0 \leqq a z \leqq z a$. Then $0 \leqq a z a \leqq z a^{2}=0$, so that $a z a=0$.

3. $(z a-a z) \notin A^{+} \cup-\left(A^{+}\right)$. Then $(z a-a z)^{+}>0$ and $(z a-a z)^{-}>0$. Now $0 \leqq(z a-a z)^{+}(z a-a z)^{-}=(z a-a z)^{+}(a z-z a)^{+} \leqq z a^{2} z=0$. Thus $(z a-a z)^{+}(z a-a z)^{-}=0$, and hence $(z a-a z)^{-}(z a-a z)^{+}=0$ since $(z a-a z)^{+} \wedge(z a-a z)^{-}=0$. Now $(z a-a z)^{+} y(z a-a z)^{-}$is a positive nilpotent of index 2 for any $y \in A^{+}$; so that, by $3.5, a(z a-a z)^{+} y(z a-$ 
$a z)^{-}=0$. Since $A$ is a prime $l$-ring and $(z a-a z)^{-}>0$, we know that $a(z a-a z)^{+}=0$ by 2.5. Similiarly, $a(z a-a z)^{-}=0$. Consequently, we have that $\left.0=a_{\imath}^{\Gamma}(z a-a z)^{+}-(z a-a z)^{-}\right]=a(z a-a z)=a z a$ for all $z \in A^{+}$. Again using 2.5, it follows that $a=0$.

Now let $a, b \in A^{+}$with $a b=0$. Then for any $z \in A^{+}, b z a$ is a nilpotent of index 2 and hence is 0 . Thus, by $2.5, a=0$ or $b=0$; and the proof is complete.

REMARK. We do not know if every prime $l$-ring $A$ in which the square of every element is positive satisfies: $a, b \in A, a \wedge b=0$, and $a b=0$ imply $b a=0$.

THEOREM 3.7. Let $A$ be an l-ring in which the square of every element is positive, suppose that disjoint elements of $A$ commute, and suppose that $A$ has zero l-radical. Then $A$ is a subdirect union of l-domains in which all squares are positive and disjoint elements commute.

Proof. B 2.14, $A$ is a subdirect union of a family $\left\{A_{\alpha} ; \alpha \in \Gamma\right\}$ of prime $l$-rings. Since both of the properties of disjoint elements commuting and all square being positive are preserved under homomorphisms, each $A_{\alpha}$ has these properties and hence is an $l$-domain by 3.6 .

COROLlary 3.8. Let $A$ be an l-ring in which the square of every element is positive, and suppose that disjoint elements of $A$ commute. Then $P(A)=\{x \in A:|x|$ is nilpotent $\}$. Moreover, if $A$ has an identity element 1 , then $P(A)=\{x \in A: x$ is nilpotent $\}$.

Proof. Since $P(A / P(A))$ is zero, $A / P(A)$ is a subdirect union of $l$-domains by 3.7. It follows that $A / P(A)$ has no nonzero positive nilpotents, and hence all of the positive nilpotents of $A$ are in $P(A)$. The first part of the corollary now follows since $P(A)$ is a nil $l$-ideal.

Finally, if $A$ has a positive identity 1 , then every nilpotent of $A$ is contained in the sub- $f$-ring $B(A)=\{x \in A:|x| \leqq n 1$ for some nonnegative integer $n\}$ of $A$ by 3.3. But an element of an $f$-ring is nilpotent if and only if its absolute value is. Thus, by the first part, $P(A)=\{x \in A: x$ is nilpotent $\}$.

THEOREM 3.9. Let $A$ be an archimedean l-ring in which the square of every element is positive. Then

(i) if $x \in A^{+}$and $x^{2}=0$, then $x A=A x=\{0\}$;

(ii) every positive nilpotent of $A$ has index $\leqq 3$;

(iii) $P(A) A^{2}=A^{2} P(A)=P(A)^{3}=\{0\}$;

(iv) $N(A)=P(A)=\{x \in A:|x|$ is nilpotent $\}$; 
(v) if $A$ has no nonzero positive left or right annihilators, then $A$ has no nonzero positive nilpotents; and

(vi) if $A$ has an identity element 1, then $A$ has no nonzero nilpotents.

Proof. The proof is broken up into several steps.

(1) If $x \in A^{+}$and $x^{2}=0$, then $x A=A x=\{0\}$.

Proof. Let $y \in A^{+}$, and let $n$ be an integer. Then $0 \leqq(n x-y)^{2}=$ $n^{2} x^{2}-n x y-n y x+y^{2}$; and hence $n(x y+y x) \leqq y^{2}$. Since $A$ is archimedean, $x y+y x=0$. Since $x y$ and $y x$ are positive, $x y=y x=0$. Since every element of $A$ is the difference of two positive elements, $x A=A x=\{0\}$.

(2) Every positive nilpotent of $A$ has index $\leqq 3$.

Proof. Let $x$ be a positive nilpotent of index $n \geqq 4$. Then $2 n-4 \geqq n$, so that $\left(x^{n-2}\right)^{2}=0$. Hence, by $(1), x^{n-1}=x\left(x^{n-2}\right)=0$; and the result follows.

(3) Let $\eta(A)=\{x \in A:|x|$ is nilpotent $\}$. Then $N(A)=P(A)=\eta(A)$.

Proof. Let $x \in \eta(A)$. For $y \in A^{+}$and $n$ an integer, we have that $0 \leqq(n|x|-y)^{2}=n^{2}|x|^{2}-n|x| y-n y|x|+y^{2} ;$ so that $n(|x| y+$ $y|x|) \leqq n^{2}|x|^{2}+y^{2}$. But $|x|^{3}=0$ by (2), so that $|x|^{2}$ is both a left and right annihilator of $A$ by (1). Hence for $z \in A^{+}$we have that $(|x| y z+$ $y|x| z) \leqq y^{2} z$. Since $A$ is archimedean, it follows that $|x| y z=y|x| z=$ 0 ; and; hence $|x| y z=y|x| z=0$ for all $y, z \in A$. Since $y|x| z=0$ for all $y, z \in A$, we have that $x \in N(A)$; and hence

$$
N(A) \subseteq P(A) \subseteq \eta(A) \subseteq N(A) \text {. }
$$

Note that since $|x| y z=0$ and $\eta(A)=P(A)$, we have that $P(A) A^{2}=$ $P(A)^{3}=\{0\}$. Moreover, if the inequality $n(|x| y+y|x|) \leqq n^{2}|x|+y^{2}$ is multiplied on the left by $z \in A^{+}$, then it follows that $A^{2} P(A)=\{0\}$. We have now completed the proofs of parts (i) through (iv).

Part (v) is an immediate consequence of part (i); and part (vi) follows from part (i) and (v) since if $A$ has an identity element, then $x$ is nilpotent if and only if $|x|$ is.

4. Subdirect unions of totally-ordered rings with no nonzero divisors of zero. In this section we prove the theorem mentioned in the introduction. It is a consequence of the following three propositions.

Proposition 4.1. Let $A$ be an $l$-ring which satisfies the identity $x^{+} a x^{-}=0$. Then an $l$-ideal $P$ of $A$ is prime if and only if $A / P$ is totally-ordered with no nonzero divisors of zero.

Proof. If $A / P$ has no nonzero divisors of zero, then $P$ is a prime $l$-ideal by 2.4 . 
Conversely, we may suppose that $A$ is a prime $l$-ring since the identity $x^{+} a x^{-}=0$ is preserved under homomorphisms. But if $x^{+} a x^{-}=0$ for all $a \in A^{+}$, then either $x^{+}=0$ or $x^{-}=0$ by 2.5. It follows that $A$ is totally-ordered. By 2.2, $A$ has no nonzero divisors of zero.

In the next proposition we shall call an $l$-ring in which $a(b \vee c)=$ $a b \vee a c$ and $(b \vee c) a=b a \vee c a$ for $a \geqq 0$ a distributive $l$-ring. Note that a distributive $l$-ring also satisfies $a(b \wedge c)=a b \wedge a c$ and $(b \wedge c) a=$ $b a \wedge c a$ for $a \geqq 0$.

Proposition. Let $A$ be a distributive $l$-ring. Then an $l$-ideal $P$ of $A$ is prime if and only if $A / P$ is totally-ordered with no nonzero divisors of zero.

Proof. Sufficiency is a restatement of 2.4 .

Conversely, let $P$ be a prime $l$-ideal of $A$. Since $A / P$ is a distributive $l$-ring, we may assume that $A$ is a prime $l$-ring. If $a \in A^{+}$is either a left or right annihilator, then $a A^{+} a=\{0\}$; so that, since $A$ is a prime $l$-ring, $a=0$ by 2.5. But ([1], Th. 14) a distributive $l$-ring with no nonzero left or right positive annihilators is an $f$-ring. Hence $A$ is totally-ordered with no nonzero divisors of zero by 2.2 .

Proposition 4.3. Let $A$ be an $l$-ring which satisfies the identity $x^{+} x^{-}=0$. Then an $l$-ideal $P$ of $A$ is prime if $A / P$ is totally-ordered with no nonzero divisors of zero.

Proof. Sufficiency is a restatement of 2.4.

Conversely, we may assume that $A$ is a prime $l$-ring since the identity $x^{+} x^{-}=0$ is preserved under homomorphisms. Then ([1], p. 59, Lemma 2) all squares of $A$ are positive. Also, disjoint elements of $A$ commute since $x^{+} x^{-}=0$ for all $x \in A$. Thus, by 3.6, $A$ is an $l$-domain. Since $x^{+} x^{-}=0$ for all $x \in A$, it follows that $A$ is totally-ordered; and hence $A$ has no nonzero divisors of zero by 2.2 .

Theorem 4.4. Let $A$ be an l-ring with zero l-radical. Then the following are equivalent:

(i) $A$ is an f-ring;

(ii) $A$ is a subdirect union of totally-ordered rings with no nonzero divisors of zero;

(iii) $x^{+} a x^{-}=0$ for all $x, a \in A$;

(iv) if $a, b, c \in A$ with $a \geqq 0$, then $a(b \vee c)=a b \vee a c$ and $(b \vee c) a=$ $b a \vee c a$; and

(v) $x^{+} x^{-}=0$ for all $z \in A$.

Proof. The equivalence of (i) and (ii) was proved by Pierce ([1], 
Th. 4) Also see Johnson [3](Theorem I. 4.8).

Since (iii), (iv), and (v) hold in any totally-ordered ring and are preserved under the formation of subdirect unions, it is clear that (i) implies (iii), (i) implies (iv), and (i) implies (v).

Now let $A$ be an $l$-ring with zero $l$-radical. Then, by $2.14, A$ is subdirect union of a family $\left\{A_{\alpha}: \alpha \in \Gamma\right\}$ of prime $l$-rings. If $A$ satisfies (iii) [(iv), (v)], then each $A_{\alpha}$ satisfies (iii) [(iv), (v)] since (iii) [(iv), (v)] is preserved under homomorphisms. By Proposition 4.1[4.2, 4.3], each $A_{\alpha}$ is totally-ordered with no nonzero divisors of zero, and the proof is complete.

The following corollary of 4.4 answers affirmatively the question of Birkhoff and Pierce originally asked in [1].

CoRollary 4.5. Let $A$ be an l-ring with an identity element 1, and suppose that $A$ has zero l-radical. Then $A$ is an f-ring if and only if 1 is a weak order unit.

Proof. Since ([1], Th. 15) 1 is a weak order unit if and only if $x^{+} x^{-}=0$ for all $x \in A$, the corollary follows from the equivalence of (i) and (v) above.

Finally we note

CoRollary 4.6. Let $A$ be an l-ring which satisfies either (iii), (iv), or (v) of 4.4. Then $P(A)=\{x \in A: x$ is nilpotent $\}$.

Proof. $A / P(A)$ is a subdirect union of totally-ordered rings with no nonzero divisors of zero. Hence all of the nilpotents of $A$ are in $P(A)$. Since $P(A)$ is a nil $l$-ideal, the corollary follows.

\section{REFERENCES}

1. G. Birkhoff and R. S. Pierce, Lattice-ordered rings, An. Acad. Brasil. Ci. 28 (1956,) 41-69.

2. N. Jacobson, Structure of Rings, Colloquium Publication no. 37, Amer. Math. Soc., Providence, 1956.

3. D. G. Johnson, A structure theory for a class of lattice-ordered rings, Acta Math. 104 (1960), 163-215.

4. N. H. McCoy, Prime ideals in general rings, Amer. J. Math. 46 (1949), 823-833.

5. R. S. Pierce, Radicals in function rings, Duke Math J. 23 (1956), 253-261.

Received January 9, 1967. This research was supported by the National Science Foundation and is part of the author's doctoral dissertation written at Purdue University under the direction of Professor Melvin Henriksen.

The Tulane University of Louisiana 


\section{PACIFIC JOURNAL OF MATHEMATICS}

\section{EDITORS}

H. ROYDEN

Stanford University

Stanford, California

\section{J. P. Jans}

University of Washington

Seattle, Washington 98105

\section{J. DUGUNDJI}

Department of Mathematics University of Southern California Los Angeles, California 90007

\section{RICHARD ARENS}

University of California

Los Angeles, California 90024

\section{ASSOCIATE EDITORS}

E. F. BECKENBACH

B. H. NEUManN

F. WOLF

K. YosIDA

\section{SUPPORTING INSTITUTIONS}

UNIVERSITY OF BRITISH COLUMBIA CALIFORNIA INSTITUTE OF TECHNOLOGY UNIVERSITY OF CALIFORNIA MONTANA STATE UNIVERSITY UNIVERSITY OF NEVADA NEW MEXICO STATE UNIVERSITY OREGON STATE UNIVERSITY UNIVERSITY OF OREGON OSAKA UNIVERSITY UNIVERSITY OF SOUTHERN CALIFORNIA
STANFORD UNIVERSITY

UNIVERSITY OF TOKYO UNIVERSITY OF UTAH WASHINGTON STATE UNIVERSITY UNIVERSITY OF WASHINGTON AMERICAN MATHEMATICAL SOCIETY CHEVRON RESEARCH CORPORATION TRW SYSTEMS NAVAL WEAPONS CENTER 


\section{Pacific Journal of Mathematics}

\section{Vol. 25, No. $1 \quad$ September, 1968}

Glen Eugene Bredon, Cosheaves and homology................... 1

Robin Ward Chaney, A chain rule for the transformation of integrals in

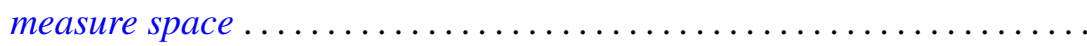

Colin W. Clark, On relatively bounded perturbations of ordinary differential operators................................... 59

John Edwin Diem, A radical for lattice-ordered rings.............. 71

Zeev Ditzian, On a class of convolution transforms ................ 83

Dennis Garoutte and Paul Adrian Nickel, A note on extremal properties characterizing weakly $\lambda$-valent principal functions............. 109

Shwu-Yeng Tzeng Lin, Fixed point properties and inverse limit spaces . . . 117

John S. Lowndes, Some dual series equations involving Laguerre polynomials ................................. 123

Kirti K. Oberai, Sum and product of commuting spectral operators ....... 129

J. N. Pandey and Armen H. Zemanian, Complex inversion for the generalized convolution transformation..................... 147

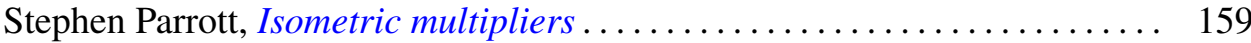

Manoranjan Prasad, Note on an extreme form .................. 167

Maciej Skwarczyński, A representation of a bounded function as infinite product in a domain with Bergman-Shilov boundary surface ......... 177

John C. Taylor, The Šilov boundary for a lattice-ordered semigroup ...... 185 Donald Reginald Traylor and James Newton Younglove, On normality and pointwise paracompactness ............................. 193

L. Tzafriri, Quasi-similarity for spectral operators on Banach spaces ... 